\title{
Dynamical Wilson twisted mass fermions: A scaling analysis
}

\section{Ines Wetzorke*, Karl Jansen, Andrea Shindler, Urs Wenger}

NIC, Platanenallee 6, 15738 Zeuthen, Germany

E-mail: ines.wetzorke, karl.jansen, andrea.shindler, urs.wengerdesy.de

\section{Istvan Montvay, Enno E. Scholz, Naoya Ukita}

DESY, Notkestr. 85, 22607 Hamburg, Germany

E-mail: istvan.montvay, enno.e.scholz, naoya.ukitaldesy.de

\section{Federico Farchioni}

Institut für Theoertische Physik, Universität Münster, Wilhelm-Klemm-Str. 9, 48149 Münster,

Germany

E-mail: federico.farchionidesy.de

\section{Luigi Scorzato}

Institut für Physik, Humboldt-Universität zu Berlin, Newtonstr. 15, 12489 Berlin, Germany

E-mail: scorzato@physik.hu-berlin.de

\section{Carsten Urbach}

NIC, Zeuthen and Institut für Theoretische Physik, Freie Universität Berlin, Arnimallee 14, 14195 Berlin, Germany

E-mail: urbach@physik.fu-berlin.de

This talk is based on reference [1] and is one of four contributions about $N_{f}=2$ dynamical twisted mass fermions which are summarized in ref. [2]. This common contribution covers three different gauge actions, namely the standard Wilson plaquette gauge action investigated here, the DBW2 [2,3] and the tree-level improved Symanzik [4] gauge action. In addition, first results are compared to next-to-leading order chiral perturbation theory formulae.

\section{References}

[1] F. Farchioni et al., Lattice spacing dependence of the first order phase transition for dynamical twisted mass fermions, hep-lat/0506025.

[2] F. Farchioni et al., Dynamical twisted mass fermions, PoS(LAT2005)072.

[3] N. Ukita et al., Scaling test of dynamical Wilson twisted mass fermions with DBW2 gauge action, PoS(LAT2005)037.

[4] U. Wenger et al., Lattice QCD with $N_{f}=2$ light Wilson fermions: the phase structure and scaling, PoS(LAT2005)044.

XXIIIrd International Symposium on Lattice Field Theory

25-30 July 2005

Trinity College, Dublin, Ireland

\footnotetext{
${ }^{*}$ Speaker.
} 